\title{
Liver Lobe
}

National Cancer Institute

\section{Source}

National Cancer Institute. Liver Lobe. NCI Thesaurus. Code C49579.

Any of the four large divisions of the liver. 